\title{
The Critical Role of the Cytoskeleton in the Pathogenesis of Giardia
}

\author{
Christopher Nosala $^{1} \cdot$ Scott C. Dawson ${ }^{1}$
}

Published online: 8 October 2015

(C) Springer International Publishing AG 2015

\begin{abstract}
Giardia lamblia is a flagellated parasite of the gut and causes significant morbidity worldwide. Novel druggable targets are sorely needed due to Giardia's prevalence and the growing threat of antibiotic resistance. Giardia's conserved and unique cytoskeletal features, such as its eight flagella and ventral disc, are required for host colonization by facilitating motility, attachment, and cell division. Therapies that target these processes could interfere with trophozoite colonization, reduce the time or severity of the infection, and reduce the number of infectious cysts shed into the environment. This requires vetting and prioritizing critical cellular processes and identifying specific Giardia proteins in those processes as targets. It is time to leverage the wealth of data gathered through genome sequencing and proteomic studies, and new insights on the cytoskeleton of Giardia to design effective new drugs to treat giardiasis.
\end{abstract}

Keywords Giardia · Gut pathogen · Microtubule · Motility · Attachment · Drug targets

This article is part of the Topical Collection on Parasitology

Scott C. Dawson

scdawson@ucdavis.edu

Christopher Nosala

cnosala@ucdavis.edu

1 Department of Microbiology and Molecular Genetics, University of California Davis, One Shields Avenue, Davis, CA 95616, USA

\section{Introduction}

Giardia lamblia is one of the most common protozoan causes of diarrheal disease worldwide $[1,2 \bullet, 3]$. The parasite belongs to the diplomonad group of microbial eukaryotes, defined by having two nuclei and eight flagella $[4,5]$. The impact of Giardia on human health is global—over one billion people currently have acute or chronic giardiasis, with rates approaching $90 \%$ in endemic areas [6, 7]. Giardia can also have an economic impact through the infection of farm animals and pets $[8,9]$. Due to the high number of giardiasis cases worldwide and the comparative lack of research efforts toward prevention and treatment, Giardia was placed on the WHO's Neglected Tropical Diseases Initiative in 2004 [1, 10, 11]. Despite its global importance, fundamental biological questions about Giardia remain and we know little about in vivo infection dynamics and pathogenesis.

Giardiasis is a zoonotic disease with a wide range of mammalian hosts that serve as reservoirs for human infection [12, 13]. Giardia parasites have a dimorphic life cycle in all hosts (Fig. 1). The dormant cyst is resistant to UV-induced light damage and changes in water tonicity [14]. Cysts are commonly acquired from contaminated water sources in regions with poor water sanitation or from lakes and streams frequented by hikers [1, 14]. Ingestion of as little as ten cysts is reported to be sufficient for robust infection [15]. During passage through the gastrointestinal tract, cysts are exposed to changes in $\mathrm{pH}$ and the presence of bile and then "excyst" to become motile trophozoites. Using flagellar motility, trophozoites navigate the lumen of the small intestine until they encounter a suitable place for attachment to the intestinal microvilli [11]. Attachment, mediated by a suction cup-like cytoskeletal structure called the ventral disc, allows the parasite to resist peristaltic flow [16]. Giardia is an extracellular parasite; cells remain in the lumen and prefer the small intestine for 


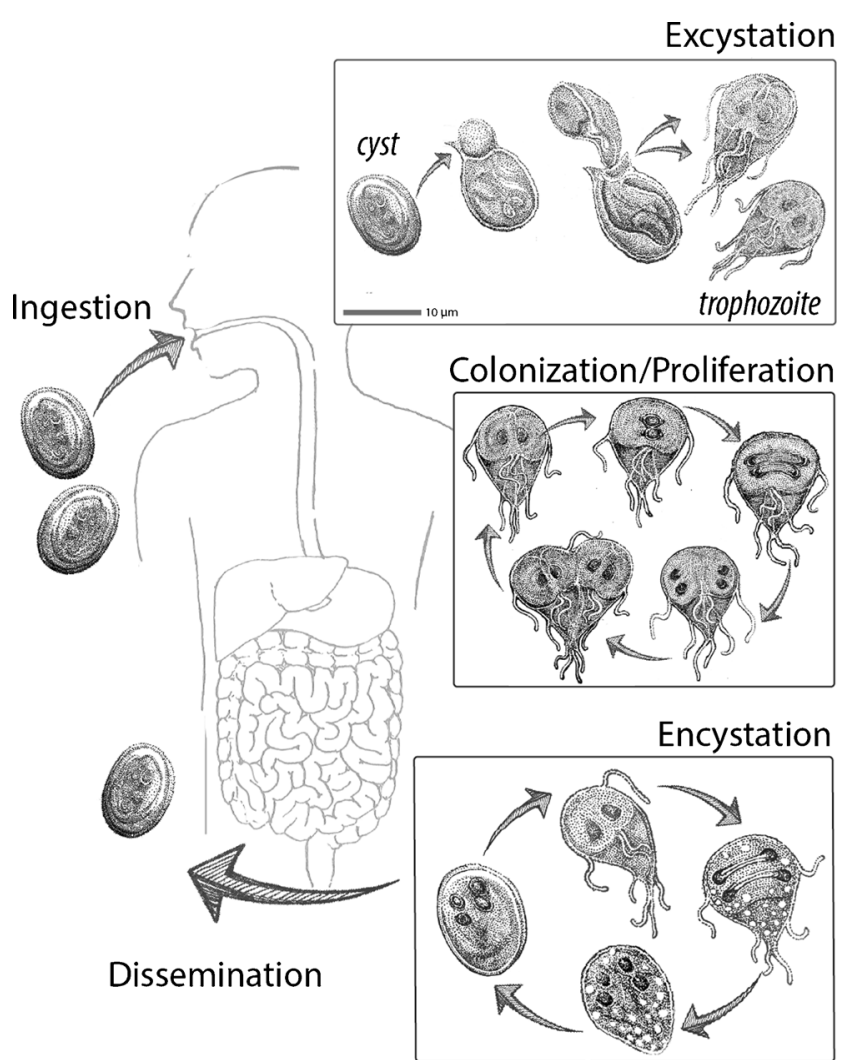

Fig. 1 The Giardia life cycle in the human host. Giardia has two life cycle stages: the flagellated trophozoite that attaches to the intestinal microvilli and an infectious cyst that persists in the environment. Cysts are ingested by the host and excyst after transiting through the stomach. Excysting trophozoites complete cell division, and then trophozoites search for suitable sites for intestinal attachment and colonization using flagellar motility. To proliferate in the small intestine and resist peristaltic flow, trophozoites attach to the villi using the ventral disc and only briefly detach to complete cytokinesis. Trophozoites thus attach, divide, and proliferate and colonize the small intestine. Based on environmental cues, partially divided trophozoites encyst and mature cysts are shed in feces. This facilitates the dissemination of parasites and the infection of new hosts. Giardia is zoonotic, and this life cycle occurs in all mammalian hosts

colonization. By factors not entirely defined, Giardia trophozoites are eventually induced to encyst and new infectious cysts exit the host via the feces. Trophozoites are also often detected in stool by antigen testing, PCR, or via microscopic examination due to their characteristic "tear drop" shape and two nuclei [17].

\section{Giardia Colonizes and Proliferates in the Mammalian Small Intestine}

The mechanism by which Giardia colonization of the gastrointestinal tract induces diarrheal disease is unclear. Giardiasis may be either acute and/or chronic and Giardia infection is generally accompanied by abdominal cramps, gas, nausea, and weight loss. Giardiasis may also result in a severe form of malabsorptive diarrhea presenting as a fatty, watery stool.
Giardiasis is especially problematic in children because it promotes malnutrition and, in some cases, affects both mental and physical development [2•]. At the cellular level, Giardia colonization of the host is known to result in villus shortening, enterocyte apoptosis, and intestinal barrier dysfunction [18]. Giardia has no known toxin and infection does not induce a robust inflammatory response, although $\mathrm{T}$ cells may be important for clearance [19]. The Giardia genome does encode over 300 variant surface protein (VSP) genes, and antigen switching of (VSPs) likely contributes to the evasion of immune screening [20,21]. Giardiasis is most commonly treated with metronidazole (Flagyl); yet, metronidazole may induce side effects including nausea and vomiting. The failure rates for anti-giardial treatment are estimated to approach $20 \%$ and symptoms sometimes return following anti-giardial treatment [7]. Furthermore, strains have been described that resist antibiotic treatment $[6,22,23]$, emphasizing the need for new therapies for the future.

\section{The Giardia Cytoskeleton Is Critical for Parasite Motility, Host Attachment, Proliferation, Encystation and Excystation, and Dissemination}

Giardia's complex microtubule (MT) cytoskeleton, including its ventral disc and flagella, is of critical importance throughout both stages of its life cycle [14,24]. Flagellar motility may play a mechanical role in the initial opening of the cyst, in addition to contractile or other MT-mediated forces [25, 26], and is also required for positioning of the trophozoite prior to attachment. The ventral disc, a spiral microtubule array, mediates trophozoite attachment via an as-yet-unknown mechanism.

Giardia cytoskeletal structures are comprised of either microtubule or actin filaments that are both intrinsically dynamic polymers that facilitate many aspects of its parasitic lifestyle [24, 27, 28]. Microtubule dynamics can be influenced by microtubule-associated proteins (MAPs), such as EB1, and microtubule-based molecular machines, such as kinesins $[29,30]$. Kinesins are dynamic motors that move along microtubules and have diverse functions in the flagellated trophozoite, ranging from flagella-mediated motility to spindlemediated chromosome segregation [31], and even to host attachment via the ventral disc. The Giardia genome contains homologs of dynein motor proteins and members of all kinesin families [32] as well as novel "hypothetical proteins" that lack homology to known MAPS [4]. The cellular roles of these novel Giardia MAPS in modulating microtubule dynamics are only beginning to be uncovered; yet, many are associated with the ventral disc or flagella [33••, 34]. While microtubule-based mechanisms of flagellar motility and mitosis are similar to those in model eukaryotic experimental systems, Giardia-specific cytoskeletal mechanisms, including ventral disc-mediated surface attachment and excystation/ 
Fig. 2 Cytoskeletal elements in Giardia with localizations of several novel, or hypothetical, proteins. The Giardia trophozoite, visualized from the ventral side using differential interference microscopy (DIC), is shown in (a). The characteristic teardrop shape is visible along with the ventral disc $(v d)$ and the four flagellar pairs $(a f l=$ anterior, $p f l=$ posteriolateral, $v f l=$ ventral, $c f l=$ caudal). Scale bar $=5 \mu \mathrm{m}$.

(b) The schematic representation of the primary elements of the trophozoite microtubule cytoskeleton shows the ventral disc $(v d)$, the median body $(m b)$, the four flagellar pairs (afl, cfl, vfl, $\mathrm{pfl})$, and the two nuclei $(n)$. The basal body for each flagellum is located between the two nuclei. In (c), DIC and fluorescent imaging of C-terminal GFP fusions demonstrates the localization of four representative proteins in Giardia localizing to the nuclei, ventral disc, flagella, or plasma membrane
A

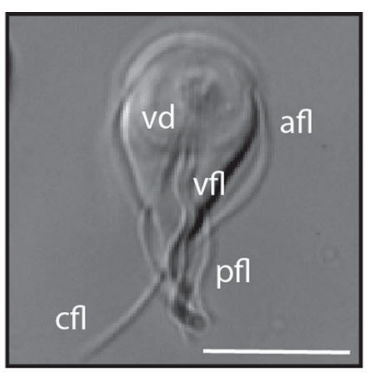

C

\section{nuclei}

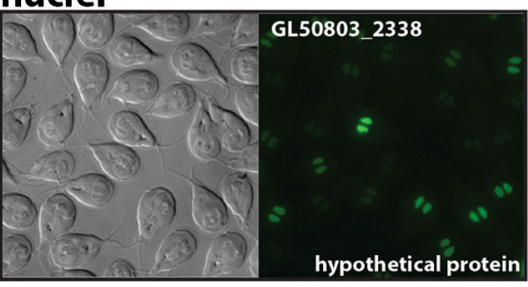

flagella

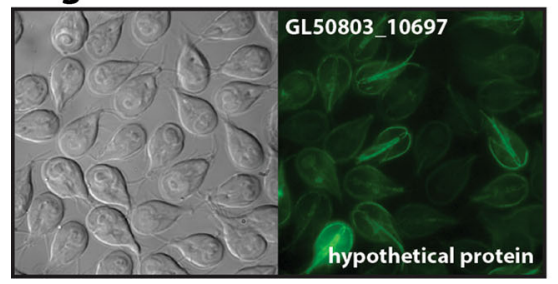

B

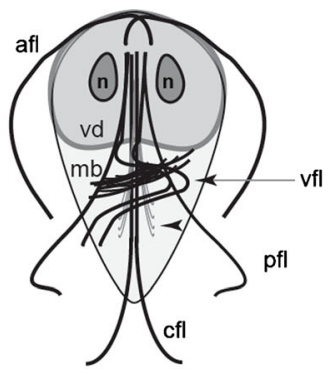

\section{ventral disc}

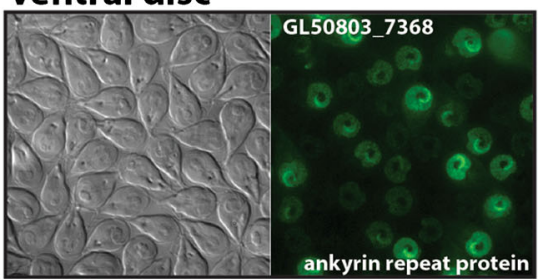

plasma membrane

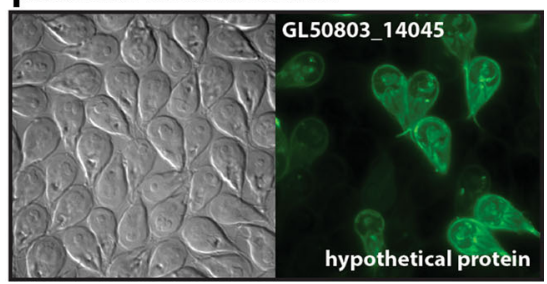

encystation, are less understood. Diverse and novel Giardiaspecific MAPs comprise Giardia microtubule structures such as the ventral disc and likely regulate and modulate Giardia microtubule dynamics in key life cycle transitions.

Giardia lacks canonical actin-associated proteins, and actin-based cellular functions have only recently begun to be described [35••]. Like microtubule-associated proteins (MAPs), actin-associated proteins also modulate actin polymerization behavior. Actin is a highly conserved protein among diverse eukaryotes. Giardia has a single copy actin gene sharing only $58 \%$ sequence identity to that of other eukaryotes [35••]. The self-assembly of globular actin (Gactin) into filaments (F-actin) promotes the hydrolysis ATP to ADP in the filament. Generally, F-actin filaments arrange into bundles and then into networks that are held together by actin cross-linking proteins promoting actin-based motility. Although Giardia lacks canonical actin-associated proteins, Giardia actin forms filaments and discrete cell-cycledependent structures and performs critical functions in trophozoites including membrane trafficking, cytokinesis, and cell morphogenesis [28, 35••, 36]. New Giardia-specific actinbinding proteins were recently identified by immunoprecipitation with Giardia actin [37]. The conserved and possibly novel mechanisms by which Giardia-specific actin-binding proteins regulate the cellular functions associated with actin likely offer promising new targets for anti-giardial therapies.

\section{Flagellar Motility Is Essential for All Stages of the Giardia Life Cycle}

Giardia trophozoites have eight flagella that are organized into four laterally bisymmetric pairs (anterior, caudal, posteriolateral, ventral). They enable cell motility and cell division, and may also be important for life cycle morphological transitions between encystation and excystation [38••, 39]. Parasite motility is a crucial process for initiating and maintaining infection in the gut, permitting the parasite to avoid peristaltic flow, and consequently search for suitable sites to attach to the intestinal lumen. Flagellar structure is conserved in trophozoites, as evidenced by the canonical " $9+2$ " microtubule formation containing radial spokes, dynein arms, nine outer doublet, and central pair microtubules [24, 40, 41]. Each axoneme originates at a basal body several microns in the cell interior and, thus, retains a significant cytosolic region before exiting at a ciliary pocket into a membrane-bound flagellum [42]. The four flagellar pairs do not all beat in the same waveform pattern; this complex force generation results in motion [39]. The flagellar pairs differ with respect to cellular position, extent of cytosolic and membrane-bound lengths, and each pair's association with ancillary flagellar structures (e.g., marginal plates) [42].

Each flagellar pair contributes differentially to overall trophozoite motility [39]. For example, "dorsal tail flexion," the 
bending of the posterior end of the trophozoite that may precede detachment, is associated with the funis (a microtubule structure that surrounds the caudal axonemes). Dorsal tail flexion may modify caudal flagellar beating, promoting parasite detachment from the intestinal villi [38••]. Anterior flagella modulate left-right motility, whereas both the anterior and ventral flagella modulate forward and downward movement. Free-swimming trophozoites also tumble, and this motility is the result of anterior, posteriolateral, and possibly ventral flagellar beating [39].

Each Giardia trophozoite has two diploid nuclei that must faithfully duplicate and segregate into daughter cells. Trophozoites undergo a "semi-open" mitosis, in which kinetochore microtubules emanate from spindle poles and enter the nuclei without dissolution of the nuclear membrane [43]. During mitosis, a process termed "flagellar maturation" occurs in which the eight flagella are shuffled and reapportioned, four parental flagella are segregated to each daughter cell, and four additional flagella are assembled de novo and segregated to the daughter cells [44]. Following chromosomal and flagellar separation (and de novo assembly of daughter ventral discs), flagellar beating aids in the separation of daughter cells to complete cell division. Giardia does not appear to utilize conserved actin-based machinery for cytokinesis as it lacks myosin motor proteins [35••]. Flagellar motility is required for completion of cytokinesis, however, as both expression of a dominant negative kinesin-2 (a motor protein required for flagellar assembly) and morpholino-based kinesin-2 knockdown result in an increase in "heart-shaped" pre-cytokinetic trophozoites [31, 45]. These kinesin-2 mutants are unable to complete cell division due to flagellar defects.

The contribution of flagellar motility in the trophozoite transition to cysts and subsequent excystation is not entirely clear. Intact flagella are observed beating within the completely formed cyst and are believed to play a role in the disruption of the cyst shell and subsequent exit of the trophozoite [46]. Both encystation and excystation from the cyst are promising processes for therapeutic targets. The prevention of encystation in particular may prevent dissemination of infectious cysts into the environment.

\section{Parasite Attachment to the Host Intestinal Epithelium via the Ventral Disc}

The ventral disc is a large microtubule-based structure that is unique to Giardia and facilitates the attachment of the parasite to surfaces. The mechanism by which attachment is achieved remains unclear: however, this mechanism permits the parasite to attach to the microvilli of a wide range of mammalian hosts as well as to inert surfaces such as glass or plastic [16]. The disc is composed of a right-handed spiral of parallel microtubules. The microtubule minus ends nucleate from the caudal flagellar basal bodies and microtubule plus ends terminate periodically throughout the disc. Microtubule plus ends are enriched in the disc overlap zone [47].

The ventral disc microtubules are also unique in that their plus ends are stable in interphase. Treatment of Giardia with drugs that affect microtubule polymerization dynamics such as Taxol and nocodazole have drastic effects on dynamic microtubules in the flagella but do not affect the length of ventral disc microtubules [31]. Furthermore, fluorescent recovery after photobleaching (FRAP) microscopy experiments indicate a lack of turnover of ventral disc proteins [33••]. The ventral disc remains a stable structure until its disassembly during the next round of cell division. During cell division, the parental ventral disc is disassembled and two daughter discs are assembled de novo on the dorsal side of the dividing cells [48]. This allows the parental disc to keep the dividing cell attached during mitosis and cell division.

Over 30 proteins localize to the ventral disc [33••]. Many disc proteins lack sequence homology to any known protein in other eukaryotes. In addition, the disc is enriched in NEK kinases and ankyrin repeat proteins that may facilitate protein/protein interactions. The disc is composed of four main substructures: microtubules and associated proteins, microribbons, crossbridges that connect the microribbons, and the lateral crest structure that surrounds the disc $[33 \bullet \bullet$. The microtubules form the foundation of the disc, with the trilaminar microribbons extending dorsally into cytosol. The microribbons are the largest component of the ventral disc, and microribbon proteins are among the most abundant in the Giardia cell. Microribbon proteins can self-assemble into polymers [49]. Regularly spaced crossbridges join the microribbons perpendicular to the orientation of the microtubules. Crossbridges likely stabilize the disc through joining of neighboring microribbons, yet may also have contractile features enabling overall conformational changes in the disc. Lastly, the lateral crest is a distinct repetitive structure encircling the exterior of the ventral disc $[33 \bullet \bullet, 47]$. The lateral crest contacts the surface during attachment and may enable a seal for suction-based attachment to surfaces [38・•]. Alternatively, the lateral crest may have contractile capabilities to allow the parasite to grasp a surface during attachment.

The mechanism by which Giardia attaches to surfaces or to the host epithelium remains unclear. The hypothesis termed the "hydrodynamic model" argued that beating of the ventral flagella is required to generate and maintain "suction" forces under the ventral disc by drawing in fluid surrounding the cell to pump through presumptive "channels" on the ventral side of the cell $[50,51]$. Despite prior reports, normal ventral flagellar beating and total flagellar beating are not required for in vitro attachment [38••]. Specifically, expression of a dominant negative alpha 2-annexin disrupted the normal beating of the ventral flagella. Dominant negative alpha 2-annexin 
parasites are able to initiate and maintain attachment despite challenge from normal and shear forces. Furthermore, morpholino-based knockdown of a central pair protein termed PF-16 causes defective flagellar waveform and morphology. Similarly, PF16 knockdowns are able to attach and resist both normal and shear forces [38••]. These studies reject the "hydrodynamic model" for attachment, although the contribution of flagellar motility to parasite site recognition for attachment in vivo remains to be determined.

Parasite attachment to surfaces is associated with conformation changes of the ventral disc, and occurs in four stages: (1) skimming motility along the surface, (2) contact of the lateral crest, (3) contact of the lateral shield, and finally (4) contact of the bare area, or central region of the ventral disc that lacks microtubules [38••]. Detachment occurs stepwise in the reverse order. While the exact mechanisms by which the ventral disc facilitates attachment are unknown, structural defects in the ventral disc decrease attachment efficiency [34]. Morpholino knockdown of the "median body protein," an abundant disc protein that lacks similarity to known proteins, results in incompletely formed ventral discs with a "horseshoe" shape. Cells with defective discs are incapable of forming a full lateral crest seal, and the discs appear flattened rather than domed. In vitro assays challenging these cells with both normal and shear forces indicate defects in attachment.

\section{The Potential for Anti-giardials that Target Cytoskeletal Structures and Processes}

The most commonly used classes of anti-giardial drugs are 5nitroimidazoles, such as metronidazole, and the benzimidazoles, such as albendazole [52]. Metronidazole is a prodrug that becomes toxic when reduced intracellularly; it induces a wide range of cellular defects, including doublestranded DNA breaks, covalent changes to protein structures, and increased oxidative stress. Albendazole has been shown to be poorly absorbed by human gut cells and likely targets beta - tubulin. Sequestering of beta - tubulin can decrease soluble tubulin pools making subunits unavailable for microtubule polymerization and can result in depolymerization of important cytoskeletal structures, including prevention of spindle formation during cell division. Metronidazole or albendazole alone is generally sufficient for clearance; however, reemergence of infection can occur, possibly due to overuse of this antibiotic [52]. To combat resistance, new therapeutics are being explored that expand 5-nitroimidazole activity [53] or target Hsp90 [54]. Drugs such as Aurofin (an antirheumatic) [55] and fumagillin have been found to be effective against Giardia [56].

Giardia is evolutionarily very distant from humans. This extreme sequence divergence - even in genes with human homologs_-offers unique possibilities for druggable targets. Further, much of the Giardia genome encodes "hypothetical" proteins that lack similarity to proteins in other organisms and, more importantly, to proteins in the human host [4]. The Giardia genome has about 6000 genes, with over 3500 expressed in trophozoites and 2500 expressed in cysts. In collaboration with EuPathDB, our lab has made efforts to subcellularly localize close to 1000 hypothetical proteins to gain insight into their functions (see Fig. 2). We have now classified hundreds of novel proteins by localization; yet, the ability to make gene knockouts to characterize cellular functions remains a challenge.

Because Giardia is a diploid in both nuclei [57], standard methods for gene disruption are not feasible [58]. Antisense morpholino oligonucleotides have been used to knock down Giardia proteins [31, 34, 35••, 38••, 42], however, the stability and penetrance of the morpholino knockdown phenotypes is not optimal for assessing or prioritizing target proteins in drug development. Genome-editing tools for efficient gene knockout in Giardia are critically needed to advance molecular genetic studies [58]|. Thus, adapting CRISPR/Cas9 technology to make stable knockouts and knockdowns in Giardia could dramatically advance drug development [59]. The CRISPR/ Cas9 system consists of an endonuclease (Cas9) capable of inducing double strand breaks in DNA and a guide RNA (gRNA) capable of both scaffolding Cas9 to the gRNA and targeting the complex to a desired sequence. Specific targeting is by base pairing of the gRNA sequence to the desired sequence. This method provides specific and efficient genome engineering and has revolutionized genetics in diverse organisms [60-63] including polyploid and asexual organisms [64, $65]$.

\section{Approaches to Identify and Validate Novel Drug Targets in Giardia}

Two general strategies are used in anti-microbial drug development and design: identifying candidate drugs and identifying candidate drug targets. No prior knowledge of a compound's mechanism of action is required when identifying candidate drugs - typically, the only requirement is that the drug reduces parasite viability. Once lead compounds are identified, they can be tested for efficacy and safety in animals and humans. Recent drug development strategies in Giardia have overwhelmingly focused on identifying new candidate anti-giardial drugs using high-throughput screening of large compound libraries. These screens have used both in vitro and in vivo assays that assess drug efficacy by quantifying parasite numbers or viability [56]. Candidate drug screening approaches have been used for decades to find drugs effective against Giardia. There are still relatively few therapeutic options for giardiasis, and the mechanisms of action of most drugs, including the nitroimidazoles, are still not well understood [66]. 
Druggable target identification is based on the assumption that knocking out the gene encoding a potential target has the same effect as administering a highly specific inhibitor of the target in vivo. A better understanding of the targets in Giardia that mediate key cellular processes in the host could vastly improve targeted drug design. In the targeted approach, target proteins are mutated and parasite phenotypes are evaluated using in vitro and in vivo assays. Drug design or screening is then performed to find compounds that specifically inhibit the activity of targets and adversely affect some aspect of parasite biology. The identification of novel targets has generally been deprioritized as a strategy for drug development in Giardia due to the lack of molecular tools to disrupt genes.

All approaches for anti-giardial drug development are limited by the availability of in vitro and in vivo assays of parasite biology and infection. Most screens measure viability [56, 66], but viability should not be the only measure of efficacy for a candidate drug. High-throughput candidate screens that only assay viability potentially overlook drugs that do not affect viability per se but do adversely affect infection. For example, trophozoites with motility defects might not withstand peristalsis and may also have cell division defects [38••]. Drugs inhibiting flagellar motility would likely be overlooked in in vitro viability assays. Even though non-motile cells would remain viable in vitro, they would have colonization defects in vivo. Thus, targeted methods are necessary to identify whether cellular processes beyond parasite metabolism are druggable.

\section{Conclusion}

Flagellar motility, attachment, and cell division are critical for host colonization. Therapies that target these processes could interfere with trophozoite colonization and proliferation, reducing the time or severity of the infection and reducing the number of shed cysts. Therefore, any or all of Giardia's basic cellular processes (e.g., flagellar functioning and motility, attachment, cell division and proliferation, or encystation) could be amenable to drug intervention. Furthermore, targeting more than one cellular process with single or multiple drugs might be key to limiting the duration, extent, or spread of infection, or drug resistance [67]. This requires vetting and prioritizing critical cellular processes, and identifying specific Giardia proteins in those processes as targets. We also need new in vitro and in vivo assays that quantify these processes to translate basic research to high-throughput drug screening. To design effective new drugs, we should leverage the wealth of genome sequencing and proteomic studies as well as new insights regarding the biology of the Giardia cytoskeleton.

\section{Compliance with Ethics Guidelines}

Conflict of Interest The authors declare they have no competing interests.

Human and Animal Rights and Informed Consent This article contains no studies with human or animal subjects performed by the authors.

\section{References}

Papers of particular interest, published recently, have been highlighted as:

- Of importance

•. Of major importance

1. Savioli L, Smith H, Thompson A. Giardia and Cryptosporidium join the 'Neglected Diseases Initiative'. Trends Parasitol. 2006;22:203-8.

2. Bartelt LA, Sartor RB. Advances in understanding Giardia: determinants and mechanisms of chronic sequelae. F1000 Prime Rep. 2010;7:62. Informative review emphasizing Giardia pathogenesis resulting from multiple factors that together may explain varying disease manifestations.

3. Muhsen K, Levine MM. A systematic review and meta-analysis of the association between Giardia lamblia and endemic pediatric diarrhea in developing countries. Clin Infect Dis. 2012;55 Suppl 4:S271-93.

4. Morrison HG, McArthur AG, Gillin FD, Aley SB, Adam RD, Olsen GJ, et al. Genomic minimalism in the early diverging intestinal parasite Giardia lamblia. Science. 2007;317:1921-6.

5. Simpson AG. Cytoskeletal organization, phylogenetic affinities and systematics in the contentious taxon Excavata (Eukaryota). Int J Syst Evol Microbiol. 2003;53:1759-77.

6. Ansell BR, McConville MJ, Ma'ayeh SY, Dagley MJ, Gasser RB, Svard SG, et al. Drug resistance in Giardia duodenalis. Biotechnol Adv. 2015;33:888-901.

7. Upcroft P, Upcroft JA. Drug targets and mechanisms of resistance in the anaerobic protozoa. Clin Microbiol Rev. 2001;14:150-64.

8. O'Handley RM, Olson ME. Giardiasis and cryptosporidiosis in ruminants. Vet Clin North Am Food Anim Pract. 2006;22:623-43.

9. Thompson RC, Palmer CS, O'Handley R. The public health and clinical significance of Giardia and Cryptosporidium in domestic animals. Vet J. 2008;177:18-25.

10. Bartelt LA, Lima AA, Kosek M, Penataro Yori P, Lee G, Guerrant RL. "Barriers" to child development and human potential: the case for including the "neglected enteric protozoa" (NEP) and other enteropathy-associated pathogens in the NTDs. PLoS Negl Trop Dis. 2013;7, e2125.

11. Ankarklev J, Jerlstrom-Hultqvist J, Ringqvist E, Troell K, Svard SG. Behind the smile: cell biology and disease mechanisms of Giardia species. Nat Rev Microbiol. 2010;8:413-22.

12. Sprong H, Caccio SM, van der Giessen JW, Network Z, partners. Identification of zoonotic genotypes of Giardia duodenalis. PLoS Negl Trop Dis. 2009;3:e558.

13. Lebbad M, Mattsson JG, Christensson B, Ljungstrom B, Backhans A, Andersson JO, et al. From mouse to moose: multilocus genotyping of Giardia isolates from various animal species. Vet Parasitol. 2010;168:231-9.

14. Adam RD. Biology of Giardia lamblia. Clin Microbiol Rev. 2001;14:447-75.

15. Rendtorff RC. The experimental transmission of human intestinal protozoan parasites. II. Giardia lamblia cysts given in capsules. Am J Hyg. 1954;59:209-20.

16. Friend DS. The fine structure of Giardia muris. J Cell Biol. 1966;29:317-32. 
17. Koehler AV, Jex AR, Haydon SR, Stevens MA, Gasser RB. Giardia/giardiasis - a perspective on diagnostic and analytical tools. Biotechnol Adv. 2014;32:280-9.

18. Halliez MC, Buret AG. Extra-intestinal and long term consequences of Giardia duodenalis infections. World J Gastroenterol. 2013;19:8974-85.

19. Heyworth MF. Immunological aspects of Giardia infections. Parasite. 2014;21:55.

20. Prucca CG, Lujan HD. Antigenic variation in Giardia lamblia. Cell Microbiol. 2009;11:1706-15.

21. Rivero FD, Saura A, Prucca CG, Carranza PG, Torri A, Lujan HD. Disruption of antigenic variation is crucial for effective parasite vaccine. Nat Med. 2010;16:551-557, 551p following 557.

22. Upcroft J, Samarawickrema N, Brown D, Upcroft P. Mechanisms of metronidazole resistance in Giardia and Entamoeba. Abstr Intersci Conf Antimicrob Agents Chemother. 1996;36:47.

23. Land KM, Johnson PJ. Molecular basis of metronidazole resistance in pathogenic bacteria and protozoa. Drug Resist Updat. 1999;2:289-94.

24. Elmendorf HG, Dawson SC, McCaffery JM. The cytoskeleton of Giardia lamblia. Int J Parasitol. 2003;33:3-28.

25. Feely DE. A simplified method for in vitro excystation of Giardia muris. J Parasitol. 1986;72:474-5.

26. Buchel LA, Gorenflot A, Chochillon C, Savel J, Gobert JG. In vitro excystation of Giardia from humans: a scanning electron microscopy study. J Parasitol. 1987;73:487-93.

27. Dawson SC. An insider's guide to the microtubule cytoskeleton of Giardia. Cell Microbiol. 2010;12:588-98.

28. Castillo-Romero A, Leon-Avila G, Perez Rangel A, Cortes Zarate R, Garcia Tovar C, Hernandez JM. Participation of actin on Giardia lamblia growth and encystation. PLoS One. 2009;4:e7156.

29. Good JA, Skoufias DA, Kozielski F. Elucidating the functionality of kinesins: an overview of small molecule inhibitors. Semin Cell Dev Biol. 2011;22:935-45.

30. Akhmanova A, Hoogenraad CC. Microtubule plus-end-tracking proteins: mechanisms and functions. Curr Opin Cell Biol. 2005;17:47-54.

31. Dawson SC, Sagolla MS, Mancuso JJ, Woessner DJ, House SA, Fritz-Laylin L, et al. Kinesin-13 regulates flagellar, interphase, and mitotic microtubule dynamics in Giardia intestinalis. Eukaryot Cell. 2007;6:2354-64.

32. Dawson SC, Cande WZ. Kinesin-like proteins in G. intestinalis confirm ancient gene duplications and divergences. Mol Biol Cell. 2001;12:435a

33.• Hagen KD, Hirakawa MP, House SA, Schwartz CL, Pham JK, Cipriano MJ, et al. Novel structural components of the ventral disc and lateral crest in Giardia intestinalis. PLoS Negl Trop Dis. 2011;5, e1442. Using a variety of high reolution imaging techniques, the authors showcase the complexity of the ventral disc and uncover novel proteins that may be required for disc function and biogenesis.

34. Woessner DJ, Dawson SC. The Giardia median body protein is a ventral disc protein that is critical for maintaining a domed disc conformation during attachment. Eukaryot Cell. 2012;11:292-301.

35.• Paredez AR, Assaf ZJ, Sept D, Timofejeva L, Dawson SC, Wang $\mathrm{CJ}$, et al. An actin cytoskeleton with evolutionarily conserved functions in the absence of canonical actin-binding proteins. Proc Natl Acad Sci U S A. 2011;108:6151-6. Using current biochemical and imaging methods, essential roles for the evolutionary distant Giardia actin are uncovered, despite the lack of actin binding proteins to mediate these processes.

36. Castillo-Romero A, Leon-Avila G, Wang CC, Perez Rangel A, Camacho Nuez M, Garcia Tovar C, et al. Rab11 and actin cytoskeleton participate in Giardia lamblia encystation, guiding the specific vesicles to the cyst wall. PLoS Negl Trop Dis. 2010;4:e697.

37. Paredez AR, Nayeri A, Xu JW, Krtkova J, Cande WZ. Identification of obscure yet conserved actin-associated proteins in Giardia lamblia. Eukaryot Cell. 2014;13:776-84.
38.• House S, Richter D, Pham JK, Dawson SC. Giardia flagellar motility is not directly required to maintain attachment to surfaces. PLoS Pathog. 2011;7:e1002167. Using genetic and imaging tools, the authors challenge and reject the commonly held 'hydrodynamic model' to explain how Giardia trophozoites maintain attachment to surfaces.

39. Dawson SC, House SA. Life with eight flagella: flagellar assembly and division in Giardia. Curr Opin Microbiol. 2010;13:480-90.

40. Holberton DV. Fine structure of the ventral disk apparatus and the mechanism of attachment in the flagellate Giardia muris. J Cell Sci. 1973;13:11-41.

41. Holberton DV. Mechanism of attachment of Giardia to the wall of the small intestine. Trans R Soc Trop Med Hyg. 1973;67:29-30.

42. Hoeng JC, Dawson SC, House SA, Sagolla MS, Pham JK, Mancuso JJ, et al. High-resolution crystal structure and in vivo function of a kinesin2 homologue in Giardia intestinalis. Mol Biol Cell. 2008;19:3124-37.

43. Dawson SC, Nohynkova E, Cipriano M. Cell cycle regulation and cell division in giardia, p 400. In: Svard HDLS, editor. Giardia: a model organism. Vienna: Springer; 2011.

44. Nohynkova E, Tumova P, Kulda J. Cell division of Giardia intestinalis: flagellar developmental cycle involves transformation and exchange of flagella between mastigonts of a diplomonad cell. Eukaryot Cell. 2006;5:753-61.

45. Carpenter ML, Cande WZ. Using morpholinos for gene knockdown in Giardia intestinalis. Eukaryot Cell. 2009;8:916-9.

46. Midlej V, Benchimol M. Giardia lambli a behavior during encystment: how morphological changes in shape occur. Parasitol Int. 2009;58:72-80.

47. Schwartz CL, Heumann JM, Dawson SC, Hoenger A. A detailed, hierarchical study of Giardia lamblia's ventral disc reveals novel microtubule-associated protein complexes. PLoS One. 2012;7, e43783.

48. Cèrva L, Nohynkova E. A light microscopic study of the course of cellular division of Giardia intestinalis trophozoites grown in vitro. Folia Parasitol (Praha). 1992;39:97-104.

49. Crossley R, Holberton DV. Assembly of $2.5 \mathrm{~nm}$ filaments from giardin, a protein associated with cytoskeletal microtubules in Giardia. J Cell Sci. 1985;78:205-31.

50. Holberton DV. Attachment of Giardia - a hydrodynamic model based on flagellar activity. J Exp Biol. 1974;60:207-21.

51. Hansen WR, Fletcher DA. Tonic shock induces detachment of Giardia lamblia. PLoS Negl Trop Dis. 2008;2:e169.

52. Leitsch D. Drug resistance in the microaerophilic parasite. Curr Trop Med Rep. 2015;2:128-35.

53. Miyamoto Y, Kalisiak J, Korthals K, Lauwaet T, Cheung DY, Lozano R, et al. Expanded therapeutic potential in activity space of next-generation 5-nitroimidazole antimicrobials with broad structural diversity. Proc Natl Acad Sci U S A. 2013;110:17564-9.

54. Debnath A, Shahinas D, Bryant C, Hirata K, Miyamoto Y, Hwang $\mathrm{G}$, et al. Hsp90 inhibitors as new leads to target parasitic diarrheal diseases. Antimicrob Agents Chemother. 2014;58:4138-44.

55. Tejman-Yarden N, Miyamoto Y, Leitsch D, Santini J, Debnath A, Gut $\mathrm{J}$, et al. A reprofiled drug, auranofin, is effective against metronidazole-resistant Giardia lamblia. Antimicrob Agents Chemother. 2013;57:2029-35.

56. Kulakova L, Galkin A, Chen CZ, Southall N, Marugan JJ, Zheng W, et al. Discovery of novel antigiardiasis drug candidates. Antimicrob Agents Chemother. 2014. doi:10.1128/AAC.03834-14.

57. Bernander R, Palm JE, Svard SG. Genome ploidy in different stages of the Giardia lamblia life cycle. Cell Microbiol. 2001;3:55-62.

58. Davis-Hayman SR, Nash TE. Genetic manipulation of Giardia lamblia. Mol Biochem Parasitol. 2002;122:1-7.

59. Ran FA, Hsu PD, Wright J, Agarwala V, Scott DA, Zhang F. Genome engineering using the CRISPR-Cas9 system. Nat Protoc. 2013;8:2281-308

60. DiCarlo JE, Norville JE, Mali P, Rios X, Aach J, Church GM. Genome engineering in Saccharomyces cerevisiae using CRISPRCas systems. Nucleic Acids Res. 2013;41:4336-43. 
61. Hwang WY, Fu Y, Reyon D, Maeder ML, Tsai SQ, Sander JD, et al. Efficient genome editing in zebrafish using a CRISPR-Cas system. Nat Biotechnol. 2013;31:227-9.

62. Wang H, Yang H, Shivalila CS, Dawlaty MM, Cheng AW, Zhang F, et al. One-step generation of mice carrying mutations in multiple genes by CRISPR/Cas-mediated genome engineering. Cell. 2013:153:910-8.

63. Mali P, Yang L, Esvelt KM, Aach J, Guell M, DiCarlo JE, et al. RNA-guided human genome engineering via Cas9. Science. 2013;339:823-6.
64. Bedell VM, Wang Y, Campbell JM, Poshusta TL, Starker CG, Krug 2nd RG, et al. In vivo genome editing using a high-efficiency TALEN system. Nature. 2012;491:114-8.

65. Clark KJ, Voytas DF, Ekker SC. A TALE of two nucleases: gene targeting for the masses? Zebrafish. 2011;8:147-9.

66. Tejman-Yarden N, Eckmann L. New approaches to the treatment of giardiasis. Curr Opin Infect Dis. 2011;24:451-6.

67. Goldberg DE, Siliciano RF, Jacobs Jr WR. Outwitting evolution: fighting drug-resistant TB, malaria, and HIV. Cell. 2012; 148:1271-83. 